\title{
Henderson Approach For Anchor Fixation Of The Fibular Head Avulsion Fracture
}

\author{
WeiHai Jiang ${ }^{1, a}$, Wei Sun ${ }^{2, b^{*}}$ and Shu Jing ${ }^{3, c}$ \\ ${ }^{1}$ Department of Orthopedics, Affiliated Hospital, Beihua University, Jilin, Jilin, China \\ ${ }^{2}$ Department of Neurology, Affiliated Hospital, Beihua University, Jilin, Jilin, China \\ ${ }^{3}$ Department of General Surgery, Affiliated Hospital, Beihua University, Jilin, Jilin, China \\ aemail:jiangwehai139@sina.com.com, bemail:sunwei2003jlu@sina.com, cemail:yitonglh@126.com
}

Keywords: Suture anchor;Fixation; Fracture.

Abstract. To investigate the surgical techniques and the clinical outcomes in the treating of the avulsion fracture of the fibula head by using suture anchors. From November 2008 to April 2013, 27 patients with the avulsion fracture of the fibular head were treated by open reduction and internal fixation with ligament repair by using suture anchors. There were 17 males and 10 females with average age of 37.3years (range 33-56years). Mean course was 10.2 months (range 8-15months). The avulsion fractures of fibular head have been proved by X-ray and 3-D CT before operation. The Lysholm's scoring system has been used for evaluation the clinical effects. The mean operation time was 78.5 minutes (range 40-105minutes). Intraoperative blood loss was 30-200ml (mean 100.0ml). Two patients were lost in follow-up after operation and the others had been followed up for 13 to 36 months (mean 14 months). X-ray films showed that all the fractures were fused in the last follow-up. According to the Lysholm's scoring system, the results were excellent in 17 cases, good in 6 cases, fair in 2 cases. The excellent and good rate was 92\%. It is emphasized that full attention should be given to reconstruction of lateral ligament structures as well as open reduction and international fixation in the treating of the avulsion fracture of the fibular head. The suture anchor is more easily, less injury than traditional internal fixation and needn't to remove.

\section{Introduction}

Fibular head avulsion fracture often occurred in a car accident, an athletes, dancing or drama actor, martial arts and manual workers. Powerful external forces directly or indirectly acting on the knee joint lead to knee varus result in external rotation instability and posterior instability of knee joint. Operation treatment aims to restore posterior structure stability.

\section{Patients and methods}

Between November 2008 and April 2013, 27 consecutive patients with the avulsion fracture of the fibular head were treated by open reduction and internal fixation by using suture anchors. There were 17 males and 10 females with average age of 37.3years (range 33-56years). There were 25 cases of traffic accident and 2 cases of sports injury. There was 1 case of old closed fracture and the others were fresh closed fractures. In these cases, 11cases were complicated with femoral condylar fractures, 15patients with tibial plateau fractures, 6 patients with medial meniscus and cruciate ligament injury, 2 cases with injury of prnoneal nerve. For the definite diagnosis, all the patients had completed the X-ray, three-dimensional CT and MRI examination before operation. The operation had carried out in 6-12 days according to the knee swelling situation. All cases had clear knee varus and/or rotary history of trauma. Equipment and materials were the suture anchors that the USA Johnson company produced.

\section{Surgical technique}

The surgical had carried out in lumar anesthesia combined with epidural anesthesia or general anesthesia. Take the supine position, knee flexion of 30 degrees, application of pneumatic tourniquet, 
with the Henderson approach, make a longitudinal oblique incision about $3.5 \mathrm{~cm}$ between the iliotibial band and the biceps femoris tendon, incise the skin, subcutaneous tissue and fascia with the fibular head as the center, the iliotibial band pulled to the front to expose the fracture, clean up the broken end of blood clots and blood stasis organization. The proximal end shift significantly with the the biceps femoris tendon and the fibular collateral ligaments stretch. Exploration of the fibular collateral ligament was carried, repaired if a tear or rupture occurred. Operation didn't need to expose the common peroneal nerve except the common peroneal nerve injury. It is easy to reset the fibular with the knee flexion 20 degrees to 30 degrees. The anchor is screwed into the medial cortical bone of the distal end without tension. And then accurately reduce the fracture. Fix it temporary by spot clamp. 2 shares cross knot suture fixation. The sutures repair and strengthen the upper tibiofibular ligament, femoral biceps and popliteal ligament. Passively straighten knee to confirm solid fixation. Cut off the excess suture, hemostasis, clean the incision and drainage, by each layer suture of incision, incision covered with a sterile dressing. Take the internal fixation for the treatment of femoral condyle fracture and tibial plateau fracture.

\section{Postoperative treatment}

Knee fixed with polymer materials cast or brace. Remove the drainage in 48-72h after operation. External fixation is generally for 2 weeks. Start functional exercise by the application of COM after external fixation removal. Take non steroidal drugs for 2 weeks to relieve pain and prevent heterotopic ossification. Begin the partial weight-bearing exercise at 6 week after operation

\section{Results}

The operation was successfully completed. The mean operation time was 78.5 minutes (range 40-105minutes Fig.1). Intraoperative blood loss was 30-200ml (mean 100.0ml Fig.2). All patients had no blood transfusion. 2 cases of preoperative common penoneal nerve injury recovered completely in 4 to 6 weeks. The other patients had no common peroneal nerve injury. Two patients were lost in follow-up after operation and the others had been followed up for 13 to 36 months (mean 14 months). According to the Lysholm's scoring system, the results were excellent in 17 cases, good in 6 cases, fair in 2 cases. The excellent and good rate was 92\%. All fractures healed well, and no lateral instability of knee joint, no fracture shift and anchor screw loosening phenomenon. At the time of the latest follow-up, all the fractures were confirmed bony healing in X-ray. 5 cases achieved bony healing in postoperative 6 months. The remaining 19 cases achieved bony healing in about postoperative 12 months. 1 case delayed healing because it was old close fracture. The skin incisions healed well. There were 2 cases of knee joint stiffness, 1 case of knee joint instability.

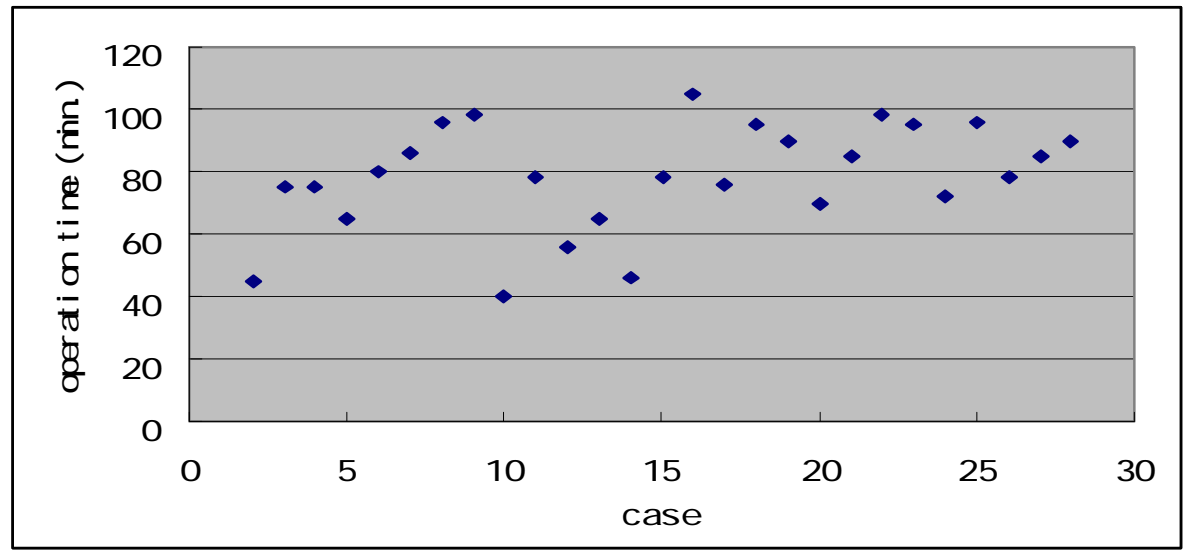

Fig.1: the operation time 


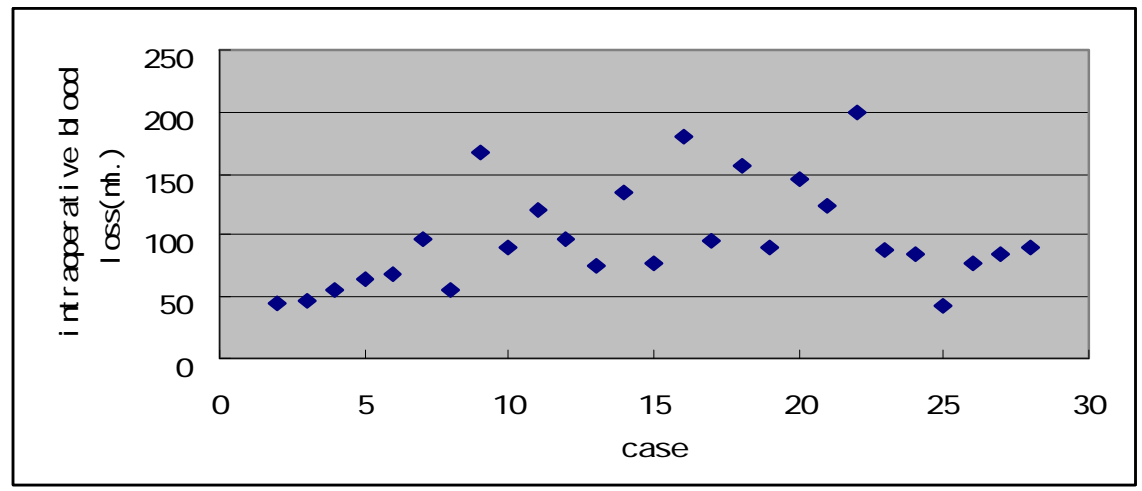

Fig.2: the intraoperative blood loss

\section{Discussion}

The Henderson approach is safer. It is far away from the common peroneal nerve. The incision is smaller. The operation time is shorter. The intraoperative blood loss is less. It is used more for meniscus injury. It is applied to fibular fracture, the effect is also very good. The proximal fibula including fibular head and fibular neck, is located in the posterolateral tibial plateau, constitutes the fretting tibiofibular joint with the lateral condyle of tibia. The femoral biceps tend, the fibular collateral ligament, popliteofibular ligament and illiotibial band parts are attached to it. The proximal fibular dislocated fracture causes the knee posterolateral complex (PLC) injury, often creates obvious posteral instability and external rotation instability ${ }^{[1-2]}$.PLC includes the popliteal muscle tendon ligament functional unit, the fibular collateral ligament, fabella fibula ligament. Popliteofibular ligament is part of the popliteal muscle-tendon-ligament functional units. Vetri ${ }^{[3]}$ applied selective ligament transaction in the study of popliteofibular ligament static effect on stability of knee joint. Vetri noted that the popliteofibular ligament and hamstring tendon tibial attachment played a role in the fight against the posterior displacement of the tibia, varus and ecternal rotation. The proximal fibular dislocated fractures are unstable, because of the pull effect of lateral collateral ligament and femoral biceps muscle. Even the fractures reduce at that time, the fractures are also very difficult to stable. This leads to nonunion of the fractures. This fracture is actually the fibular collateral ligament and the femoral biceps tendon and popliteofibular ligament injury of support structure. Such as the occurrence of nonunion of the fractures, it can lead to lateral instability of knee joint and knee flexion weakness. If the fracture position is low (located in the neck of the fibular) can also cause compression of common peroneal nerve. Therefore, when the fracture shifts, it should be surgical repaired. This can reconstruct the knee joint stability and avoid the secondary injury of common peroneal nerve and the possible fracture nonunion.

The aim of the proximal fibular fracture operation is two general purposes. One is the exploration of the common peroneal nerve injury with the proximal fibular fracture. The second is the stability reconstruction of the joint. After the reduction of the fracture, external force that leads to shift the fracture end mainly comes from the femoral biceps tendon. So, as long as the offset of the tension holding shares of biceps tendon, fracture redisplacement end possibility is greatly reduced. For this kind of fracture, the clinical application of fixation is generally Kirschner wire. Its advantage is reliable fixation and has a strong tension. Its shortcoming is that the surrounding soft tissue is stripped more and the steel wire and Kirschner wire end may damage the common peroneal nerve. Anchor fixation theory sources from the farm sinking principle of the falling support fence column on American South Texas ${ }^{[4]}$. Big rocks buried fix the fence with wires, wire and ground angle called sink angle is $45 \mathrm{deg}$. The current clinical application design of the suture anchor comes from the principle. In order to fix effectively and reduce the suture tension at the same time, the clinical selection of implantation of suture anchor angle is $45 \mathrm{deg}$. Suture anchors fixation for such fractures has the following advantages ${ }^{[5]}$. The operation is simple and convenient, shortens the operation time, no need surgery to remove it. It can deduce the soft tissue dissection, less trauma. Prees-fit fixation 
method makes it stable on the cortical bone. The Ethibond suture maintains lag for a long time in the soft tissue healing period. This can make the ligament repaired for good intensity and link closely with osteoporosis. It is suitable for the development of early postoperative functional exercise in order to prevent joint stiffness.

Two patients were lost in follow-up after operation and the others had been followed up for 13 to 36 months (mean 14 months). X-ray films showed that all the fractures were fused in the last follow-up. According to the Lysholm's scoring system, the results were excellent in 17 cases, good in 6 cases, fair in 2 cases. The excellent and good rate was 92\%. All the fractures healed well, and no lateral instability, no fracture shift and anchor screw loosening phenomenon. It benefits from the early surgical treatment of stable lateral structure of the knee joint, so that patients can functional exercise early to prevent joint stiffness. 1 case was delayed healing, that the fracture was comminuted. It is difficult to reduce the fracture fragments with the tendolysis of the femoral biceps tendon. The iliotibial tract is made of cord, cut it to required length, respectively tailed with the proximal fragment of the suture. The fracture end needn't graft bone. The knee joint function is fair by the follow-up.

\section{Conclusions}

This study shows that, with suture anchors fixation in treatment fibular head displaced fracture, the trauma is less, the fixation is reliable, patients functional exercise earlier, patients don't need surgery for remove the anchor.

\section{Acknowledgements}

Weihai Jiang and Wei Sun are the corresponding authors. The project is supported by the Science and Technology Bureau of Jilin City in China. (No. 201437012, No. 2014).

\section{References}

[1] Harner C, Hoher J, Vogrin T, et al. The effects of a popliteusmuscle load on insitu forces in the posterior cruciate ligament and on knee kinematics. A human cadaveric study. Am J Sports Med, 26 (5) pp, 669-673, 1998.

[2] DeLee J, Rlley M , Rockwood C, et al. Acute posterolateral rotatory instability of the knee . Am J Sports Med, 11 (4), pp.199-207, 1983.

[3] Veltri DM, Deng XH, TorzilliPA, et al. The role of the popliments in Stability of the human knee. A biomechanical study. Am J Sports Med, 24, pp.19-27, 1996.

[4] Burkart SS. The deadman theory of suture anchors: observation salonga South Texas fence line. Arthroscopy,11(1), pp.119, 1995.

[5] Chen Nong, Li Zhi, Dong Jian, et al. Treatment of injury of triangular ligament of ankle joint with suture anchors. China Journal of Bone and Joint Injury, 7,pp.235-236 , 2011. 